\title{
Estudo clínico dos nevos melanocíticos congênitos na criança e no adolescente*
}

\section{Clinical study of the congenital melanocytic naevi in the child and adolescent}

\author{
Nurimar Conceição Fernandes ${ }^{1}$
}

José Leonardo Rodrigues Machado²

Resumo: Fundamentos: a classificação dos nevos melanocíticos congênitos (NMC) e a magnitude do risco de transformação em melanoma são ainda polêmicos.

OвJETIVOs: Analisar o perfil dos NMC em crianças e adolescentes no IPPMG-UFRJ segundo sexo, cor, idade, tipo clínico, localização e evolução.

Material e MÉTODos: Estudo longitudinal de coorte retrospectivo e prospectivo de 1994 a 2007; amostra de demanda espontânea e referida.

RESUlTADOS: 30 crianças e 30 adolescentes apresentaram 74 NMC: 60,8\% (pequenos), 27\% (médios), $5,4 \%$ (grandes) e $6,7 \%$ (gigantes), sendo que $45,9 \%$ no sexo masculino e $54 \%$ no sexo feminino e $45,9 \%$ em brancos e $54, \%$ em não brancos. Sexo e cor não influenciaram o tipo clínico. Dentre os pequenos e médios, $27,1 \%$ apresentaram-se no tórax e $23 \%$ na cabeça e pescoço; os grandes e gigantes no pólo cefálico, região cervical, linha média posterior e membros; $28,3 \%$ foram seguidos por mais de 10 anos, $47,3 \%$ entre três e nove anos e $24,3 \%$ por tempo inferior a três anos. Os NMC pequenos e médios se mantiveram inalterados; um grande e dois gigantes mostraram clareamento; nenhum caso desenvolveu melanoma.

Conclusão: distribuição homogênea entre brancos/não brancos e sexo masculino/ feminino. O sexo e a cor não tiveram relação com o tipo clínico; os NMC pequenos predominaram com localização preferencial no tronco.

Palavras-chave: Adolescente; Criança; Melanoma; Nevo pigmentado

\begin{abstract}
BACKGROUND: The classification of the congenital melanocytic nevus (CMN) and the magnitude of the risk of transformation into melanoma are still controversal.

OвJECTIVE: To analyse the profile of the CMN in the child and teenager at IPPMG-UFRJ according to sex, skin colour, age, clinical type, locations and evolution.

METHODS: Longitudinal study of retrospective and prospective cohort from 1994 to 2007 . Aleatoric sample from spontaneous or referal demand.

Results: 30 children and 30 teenagers showed 74 nevi: 60,8\% (small), 27\% (medium), 5,4\% (large) and $6,7 \%$ (giant); from these, $45,9 \%$ were in the male sex and $54 \%$ in the female sex. There were $45,9 \%$ in white and 54\% in non-white. Sex and skin colour didn't influence the clinical type. Among the small and medium nevi, $27,1 \%$ were located in the trunk, $23 \%$ in the head and neck; the large and giant ones in the head, neck, back and limbs; 28,3\% were attended for more than 10 years, $47,3 \%$ between three and nine years and $24,4 \%$ for a time below three years; the small and medium CMN kept unchanged. The fading of the lesion was observed in one large and in two giant CMN. No cases of melanoma were observed.

ConClusion: An homogeneous distribution among white/non white and male/female; the sex and the ethnic group did not influence the clinical type; the small CMN was the most frequent clinical type mainly in the trunk.

Keywords: Adolescent; Child; Melanoma; Nevus, pigmented
\end{abstract}

Recebido em 15.5.2008.

Aprovado pelo Conselho Consultivo e aceito para publicação em 04.03 .09

* Trabalho realizado no Instituto de Puericultura e Pediatria Martagão Gesteira da Universidade Federal do Rio de Janeiro (IPPMG/UFRJ) - Rio de Janeiro (RJ), Brasil. Conflito de interesse: Nenhum / Conflict of interest: None

Suporte financeiro: Nenhum / Financial funding: None

Professora associada, Faculdade de Medicina, Universidade Federal do Rio de Janeiro (UFRJ). Hospital Universitário Clementino Fraga Filho - Instituto de Puericultura e Pediatria Martagão Gesteira - Rio de Janeiro (RJ), Brasil

Pós-graduada em Medicina (Dermatologia), Faculdade de Medicina, Universidade Federal do Rio de Janeiro (UFRJ) - Rio de Janeiro (RJ), Brasil.

CC2009 by Anais Brasileiros de Dermatologia

An Bras Dermatol. 2009;84(2):129-35. 


\section{INTRODUÇÃO}

Os nevos melanocíticos congênitos (NMCs) são morfologicamente redondos ou ovais, com bordas regulares ou irregulares e bem definidas. Frequentemente, apresentam tom marrom-claro com áreas de marrom-escuro. A superfície é papulosa, rugosa, verrucosa ou cerebriforme; de modo geral, são únicos, mas nos nevos maiores há lesões satélites; são assintomáticos e eventualmente apresentam prurido, hipersensibilidade, xerose e anidrose..$^{1,2}$

Com o tempo, adquirem pelos, tornam-se mais escuros, com tons uniformes de marrom, marromescuro ou preto ou ficam mais claros, porém raramente regridem. Fato comum entre os NMCs grandes ou gigantes é a formação de nódulos. Nos neonatos, geralmente, são mais claros e sem pelos. Alguns desenvolvem halo acrômico, com conseqüente apagamento do nevo (nevo halo). ${ }^{1,2}$

O NMC e o nevo melanocítico adquirido apresentam características histológicas semelhantes. Os seguintes aspectos auxiliam na diferenciação: no NMC, os nevomelanócitos localizam-se entre as fibras da derme reticular, nos dois terços inferiores da derme e no interior da hipoderme, bem como ao redor e no interior dos folículos pilosos, glândulas sebáceas, aparato écrino, parede de vasos e nervos. ${ }^{1} \mathrm{O}$ diagnóstico do NMC é primariamente clínico e a sua presença ao nascimento ou imediatamente após é conclusiva. ${ }^{3}$

Há muitas controvérsias na classificação dos NMCs; a que nos parece mais adequada e que a maioria dos autores adota é: NMC pequeno $(<1,5 \mathrm{~cm})$; NMC médio $(1,5 \mathrm{~cm}-10 \mathrm{~cm})$; NMC grande $(11 \mathrm{~cm}-$ $20 \mathrm{~cm})$; NMC gigante $(>20 \mathrm{~cm})$ (Figuras $1-4) .{ }^{4}$

$\mathrm{O}$ risco de transformação maligna nos NMCs, de modo geral, varia de $5 \%$ a $40 \%{ }^{1}$ e de $4,5 \%$ a $10 \%{ }^{5}$ A revisão da literatura mostra muitas controvérsias em relação ao risco em NMCs grandes/gigantes (1\%$42 \%),{ }^{6,7}$ médios $(3 \%-21 \%)^{1,8}$ e pequenos $(8,1 \%-14,9 \%) .{ }^{9}$ O desenvolvimento de melanomas em NMC grande e gigante ocorre antes da puberdade e, em nevos pequenos e médios, geralmente, após a puberdade..$^{10-13}$

Considerando que as publicações sobre NMCs são escassas em nosso meio, objetivamos analisar o seu perfil clínico na criança e no adolescente no Instituto de Puericultura e Pediatria Martagão Gesteira.

\section{MATERIAL E MÉTODOS}

No período de 1994 - 2007, crianças (0 - 9 anos) e adolescentes (10 - 19 anos) com NMC (detectado ao nascimento ou até dois anos de idade pelos pais e/ou responsáveis) foram submetidos a um estudo longitudinal de coorte retrospectivo e prospectivo. A amostra foi de demanda espontânea e referida, e os

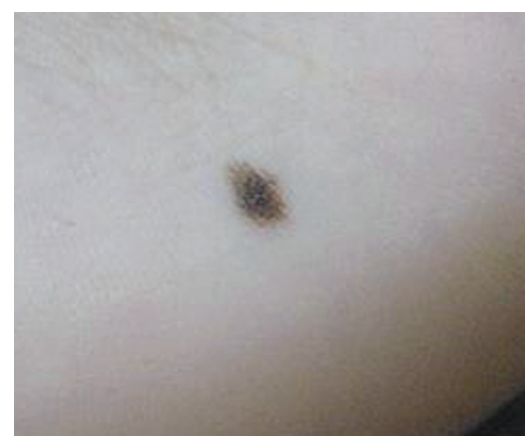

Figura 1: Nevo melanocítico congênito pequeno

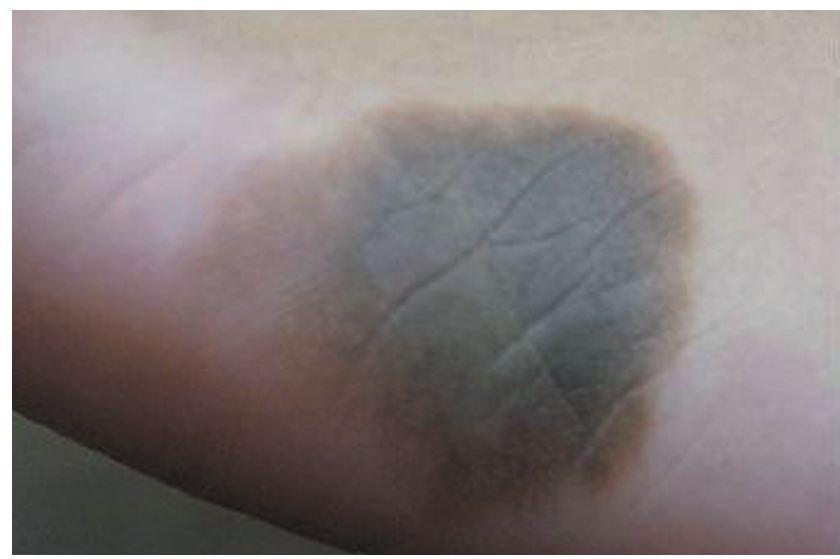

Figura 2: Nevo melanocítico congênito médio

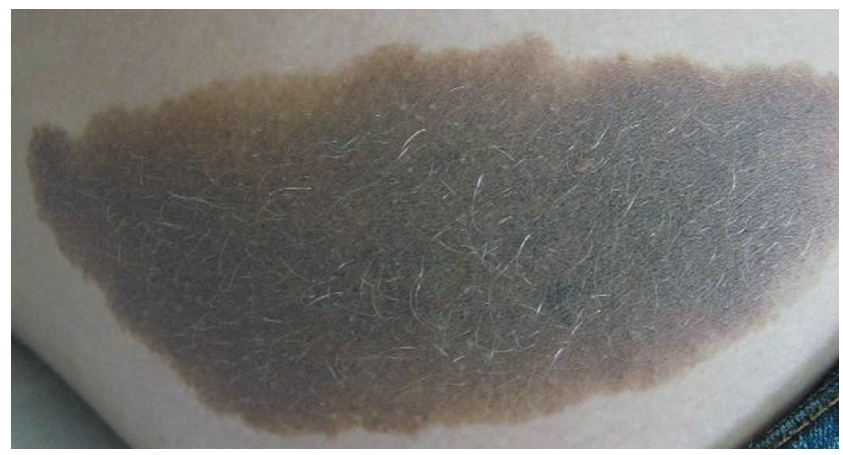

FigurA 3: Nevo melanocítico congênito grande

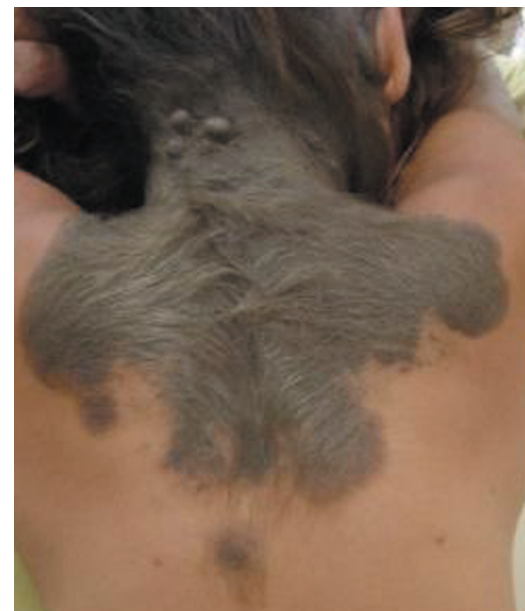

Figura 4: Nevo melanocítico congênito gigante 
NMC, queixa principal, ou detectados durante a consulta por outra queixa dermatológica. As variáveis estudadas foram idade, sexo, cor (branco / não branco), tipo clínico, localização e evolução.

\section{Seguimento ambulatorial:}

1) NMC pequeno $(<1,5 \mathrm{~cm})$ e NMC médio $(1,5$ a $10 \mathrm{~cm})$

- a cada seis meses.

2) NMC grande (11 a $20 \mathrm{~cm})$.

- de três em três meses no primeiro ano de vida e de seis em seis meses após o primeiro ano até a puberdade.

3) NMC gigante $(>20 \mathrm{~cm})$.

- de três em três meses no primeiro ano de vida e de seis em seis meses após o primeiro ano até a puberdade; palpação de fígado, baço, linfonodos. ${ }^{14}$

\section{4) Orientação para fotoproteção.}

A análise estatística foi realizada pelo teste quiquadrado ou pelo teste de Fisher, quando o primeiro não pôde ser utilizado.

\section{RESULTADOS}

Quanto às idades por ocasião da entrada no estudo, os pacientes apresentaram a seguinte distribuição:

- seis crianças entraram com um ano de idade;

- 15, aos dois anos;

- 10, aos três anos;

- nove, aos quatro anos;

- nove, aos cinco anos;

- dois, aos seis anos;

- quatro, aos sete anos;

- um, aos nove anos;

- dois adolescentes, aos 10 anos;

- um, aos 11 anos e

- um, aos 12 anos,

totalizando 56 crianças e quatro adolescentes (Tabela 1).

Considerando-se o tipo de NMC e a idade quando da entrada no estudo, verifica-se distribuição homogênea, provavelmente, pelos tipos de demanda (espontânea e/ou referida) (Tabela 1).

Em 2007, a casuística era composta por 30 crianças - seis meninos brancos/sete não-brancos; sete meninas brancas/10 não-brancas - e 30 adolescentes, sendo cinco brancos/sete não-brancos e nove brancas/nove não-brancas. No total, eram 25 casos do sexo masculino (11 brancos/14 não-brancos) e 35 casos do sexo feminino (16 brancas/19 não-brancas) (Tabela 2).
Foram observados no total 74 nevos: 45 NMCs pequenos $(60,8 \%), 20$ NMCs médios $(27 \%)$, quatro NMCs grandes $(5,4 \%)$ e cinco NMCs gigantes $(6,7 \%)$, sendo 34 (45,9\%) no sexo masculino e 40 (54\%) no sexo feminino; seis casos com dois NMCs pequenos; um caso com cinco NMCs pequenos; um caso com quatro NMCs pequenos; um caso com dois NMCs médios (Tabela 3).

Para fins de análise estatística, agruparam-se, primeiramente, os nevos grandes e gigantes; posteriormente, para uma segunda análise, agruparam-se os números de nevos médios, grandes e gigantes, pois, separadamente, seus valores não foram estatisticamente significativos. Os testes não evidenciaram relação entre as variáveis estudadas $(\mathrm{p}=0,5 ; \mathrm{p}=0,24)$ (Tabela 3).

Trinta e quatro nevos $(45,9 \%)$ em brancos e 40 nevos (54\%) em não-brancos foram observados. Para análise estatística, novamente se agruparam os valores como foi feito na tabela anterior, não sendo encontrada relação entre as variáveis $(p=0,23)$ (Tabela 4).

Dentre os nevos pequenos e médios, 18 (27,7\%) se localizavam no tórax, 15 (23\%) na cabeça e pescoço, 12 (18,4\%) no abdômen e virilha, 11 (17\%) no membro inferior, oito $(12,4 \%)$ no membro superior e um (1,5\%) na genitália (Tabela 5). Esta tabela apresenta uma grande quantidade de categorias, sendo apenas descritiva e a qual não se aplicam, portanto, os testes estatísticos. Observa-se que o tronco é a principal localização.

Três NMC grandes se localizavam na coxa e um NMC grande se localizava na mão e membro superior. Os cinco NMC gigantes se distribuíam em: 1) calção de banho; 2) coxa e membro inferior; 3) dorso, região peitoral e ombro; 4) couro cabeludo; 5) couro cabeludo, pescoço e parte superior do dorso.

Dos 74 nevos estudados, 21 (28,3\%) foram seguidos por mais de 10 anos, 35 (47,3\%), entre três e nove anos e $18(24,4 \%)$, por tempo inferior a três anos. Não foi encontrado nenhum caso de melanoma (Tabela 6 ).

Em relação à evolução, observou-se que os NMC médios se mantiveram inalterados. Um dos nevos médios em abdômen apresentava uma área de vitiligo na borda medial. Dos quatro NMCs grandes, o de localização em coxa mostrou clareamento e os três restantes não mostraram alterações. Dos cinco NMCs gigantes, dois (calção de banho e outro em coxa e membro inferior) mostraram clareamento, principalmente, em borda, e três mantiveram-se inalterados.

\section{DISCUSSÃO}

A revisão da literatura demonstra que a maioria dos estudos sobre NMC aborda apenas os grandes e gigantes. Poucos utilizaram a metodologia desta investigação, isto é, incluindo NMCs gigantes, grandes, médios e pequenos. ${ }^{11,13,15-18}$ Alguns autores discutiram risco de malignidade e conduta dos NMCs baseando-se 
TABela 1: Distribuição dos nevos melanocíticos congênitos segundo idade dos pacientes na entrada do estudo

\begin{tabular}{|c|c|c|c|c|c|}
\hline \multirow[b]{2}{*}{ NMC } & \multicolumn{4}{|c|}{ Idade na entrada do estudo / $\mathrm{N}^{\circ}$ de casos } & \multirow[b]{2}{*}{ TOTAIS } \\
\hline & Até 2 anos & $>2-3$ anos & $>3-4$ anos & $>4$ anos & \\
\hline Pequeno & 12 & 10 & 9 & 14 & 45 \\
\hline Médio & 5 & 3 & 6 & 6 & 20 \\
\hline Grande & 1 & 1 & - & 2 & 4 \\
\hline Gigante & 3 & 1 & 1 & - & 5 \\
\hline TOTAIS & 21 & 15 & 16 & 22 & 74 \\
\hline
\end{tabular}

Fonte: IPPMG-UFRJ (1994-2007).

TABela 2: Distribuição dos casos segundo a cor e o sexo

\begin{tabular}{|c|c|c|c|c|c|c|}
\hline \multirow[b]{2}{*}{ Sexo } & \multicolumn{3}{|c|}{ Cor } & \multirow[b]{2}{*}{$\%$} & \multirow[b]{2}{*}{ Total } & \multirow[b]{2}{*}{$\%$} \\
\hline & Branca & $\%$ & Não-branca & & & \\
\hline Masculino & 11 & & 14 & & 25 & 41,6 \\
\hline Feminino & 16 & & 19 & & 35 & 58,3 \\
\hline Total & 27 & 45 & 33 & 55 & 60 & 100 \\
\hline
\end{tabular}

Fonte: IPPMG/UFRJ (1994-2007).

em casuísticas exclusivas de NMCs grandes/gigantes. As controvérsias na classificação dos NMCs não sanadas ao longo do tempo e a informação sobre a época de apresentação (principalmente do NMC pequeno) são tropeços na abordagem de tais lesões. O NMC, proliferação nevomelanocítica benigna presente ao nascimento, pode não ser aparente ao exame clínico do recém-nato, devido à falta de pigmento visível (NMC tardio). Este nevo tardio desenvolve pigmento de forma lenta e, com o tempo, se torna visível, na maioria das vezes, durante os dois primeiros anos de vida e tem características clínicas e histológicas indistinguíveis do NMC típico. O critério de inclusão no estudo foi a informação obtida dos pais e/ou responsáveis sobre a época de seu aparecimento (até dois anos).

A documentação fotográfica desde o início é ideal, mas nem sempre exequível, como se percebe na literatura. No presente estudo, quando havia dúvidas na informação dos pais e/ou responsáveis, o caso era excluído. Recentemente, foi proposto que o nevus spillus integrasse o espectro dos NMCs, apesar de congênito ou adquirido. Optou-se pela não-inclusão.

A distribuição dos casos deste estudo foi homogênea entre brancos/não-brancos e sexo masculino/feminino; sexo e cor não influenciaram o tipo clínico dos nevos. Na literatura, entre 133 NMCs de diferentes tamanhos, relata-se uma relação 3:2 em relação a sexo feminino/masculino, sendo a maioria das crianças caucasianas e apenas um negro. ${ }^{15}$ Para outros autores, ${ }^{16,18}$ nenhuma diferença de significância foi observada em relação ao sexo. Em acompanhamento de 46
NMCs, observaram-se 29 do sexo feminino e 17 do sexo masculino, sendo 45 brancos e um não-branco. ${ }^{17}$

Em nossa casuística foram observados NMC pequenos (60,8\%), NMC médios (27\%), NMC grandes $(5,4 \%)$ e gigantes $(6,7 \%)$. Outros estudos relatam $7 \%$ de NMC grandes e 93\% pequenos; ${ }^{13} 28$ (10\%) de nevos gigantes; ${ }^{16} 80,3 \%$ de nevos pequenos/ médios e $19,7 \%$ de nevos grandes. ${ }^{11}$ Nosso estudo confirma a prevalência dos nevos pequenos referida na literatura.

Os NMC pequenos se localizavam predominantemente no tronco, enquanto os NMC médios se distribuíam anatomicamente de forma mais homogênea. As porcentagens referidas na literatura para as diferentes áreas do corpo são cabeça (16\%), pescoço (17\%), tórax (50\%), membro superior (7\%) e membro inferior (4\%); sem diferenças entre meninos e meninas; ${ }^{16}$ 14,6\% (tórax) e 15,2\% (abdômen). ${ }^{11}$ Em outro estudo, ${ }^{18}$ os nevos são relatados preferencialmente, no tronco e abdômen, com baixa concentração em cabeça e membros superiores. Nenhum foi encontrado em regiões palmares ou plantares, sendo únicos em $90 \%$ dos casos.

O desenvolvimento do nevo melanocítico adquirido parece estar relacionado a alguns fatores, como tipo de pele, etnia, predisposição genética e exposição à luz ultravioleta. Nevos adquiridos costumam ser mais numerosos em áreas cronicamente expostas ao sol e em crianças com pele clara, cabelos louros e olhos azuis. Isso não está descrito para os NMCs. ${ }^{19}$

Quanto ao tempo de seguimento ambulatorial, nosso estudo teve uma média de 5,7 anos, enquanto 
TABela 3: Distribuição dos nevos segundo sexo e tipo clínico

\begin{tabular}{|c|c|c|c|c|c|c|c|c|c|c|}
\hline \multirow[t]{2}{*}{ Sexo } & \multicolumn{8}{|c|}{ Tipo clínico } & \multirow[b]{2}{*}{ Total } & \multirow[b]{2}{*}{$\%$} \\
\hline & Pequeno & $\%$ & Médio & $\%$ & Grande & $\%$ & Gigante & $\%$ & & \\
\hline Masculino & 20 & & 11 & & 2 & & 1 & & 34 & 45,9 \\
\hline Feminino & 25 & & 9 & & 2 & & 4 & & 40 & 54 \\
\hline Total & 45 & 60,8 & 20 & 27 & 4 & 5,4 & 5 & 6,7 & 74 & 100 \\
\hline
\end{tabular}

TABela 4: Distribuição dos nevos segundo cor e tipo clínico

\begin{tabular}{|c|c|c|c|c|c|c|c|c|c|c|}
\hline \multirow[t]{2}{*}{ Cor } & \multicolumn{8}{|c|}{ Tipo clínico } & \multirow[b]{2}{*}{ Total } & \multirow[b]{2}{*}{$\%$} \\
\hline & Pequeno & $\%$ & Médio & $\%$ & Grande & $\%$ & Gigante & $\%$ & & \\
\hline Branca & 23 & & 6 & & 1 & & 4 & & 34 & 45,9 \\
\hline Não-branca & 22 & & 14 & & 3 & & 1 & & 40 & 54 \\
\hline Total & 45 & 60,8 & 20 & 27 & 4 & 5,4 & 5 & 6,7 & 74 & 100 \\
\hline
\end{tabular}

Fonte: IPPMG/UFRJ (1994-2007) (p=0,23).

na literatura são relatados períodos médios compreendidos dentre 3,4 e 23,7 anos. ${ }^{13,15,16,17}$

$\mathrm{Na}$ presente casuística, dentre os quatro NMCs grandes, um apresentou clareamento parcial e, entre os cinco NMCs gigantes, dois apresentaram clareamento parcial. Um estudo ${ }^{15}$ evidenciou clareamento em $17 \%$ dos NMCs grandes. Foi publicado um caso de NMC gigante $^{20} \mathrm{e}$ dois casos de nevos médios ${ }^{21}$ com regressão espontânea. Verificou-se que o clareamento não é fato incomum no NMC grande/gigante. A revisão bibliográfica mostra que o curso dos NMCs é variado: regressão pigmentar parcial ou total; halo de hipopigmentação (nevo-halo) com regressão espontânea após a formação do halo; área de vitiligo, em adição ao aparecimento do halo. A regressão pigmentar é um processo no qual os melanócitos dérmicos perdem sua capacidade de produzir pigmento, restando apenas o pigmento dos melanócitos da camada basal. Várias teorias têm sido propostas para explicar estas alterações de pigmentação: precursores naturais da melanina seriam inibidores de sua produção; sendo assim, os melanócitos perderiam seus mecanismos protetores que desabilitam estes precursores. A teoria imunológica é a mais aceita para a supressão da produção de pigmento através da ação da imunidade humoral e celular. Outra hipótese formulada é a de que agentes neurotóxicos seriam liberados próximo ou no interior dos melanócitos, destruindo as células produtoras de melanina ou simplesmente parando a sua produção. A eliminação transepidérmica das células névicas, bem como, sua degeneração gordurosa também são citadas. ${ }^{20,21}$
A média de idade de sete anos para o desenvolvimento do melanoma sobre o NMC relatada na literatura coloca o risco máximo na infância (grande/gigante) e na adolescência (médio); o risco de desenvolvimento de melanoma no NMC pequeno é apontado para a idade adulta (em torno de 60 anos).$^{2,9,22,23} \mathrm{O}$ fato de o NMC pequeno ser mais frequente do que o gigante e, muitas vezes, múltiplo permite concluir que seu potencial de malignidade deve ser de maior importância prática do que o NMC gigante. Em 204 casos de melanoma, foi observado que 44 (21,6\%) resultaram de um NMC pequeno; nenhum caso ocorreu antes dos 20 anos e não houve faixa etária preferencial de transformação. ${ }^{23} \mathrm{O}$ desenvolvimento de melanoma não foi observado em 230 casos de NMC de tamanho médio acompanhados até a idade de 25 anos. ${ }^{22}$ Outros autores não referem transformação entre 3.922 NMCs pequenos/médios acompanhados por dez $\operatorname{anos}^{13}$ e 164 NMCs pequenos durante 25 anos. ${ }^{16}$ Para o NMC grande, é apontado o percentual de transformação de $3,8 \%{ }^{4}$

Não evidenciamos nenhum caso de malignização. A literatura consultada refere que os NMCs grandes/gigantes apresentam maior risco para desenvolvimento de melanomas e que o risco dos NMCs médios e pequenos continua controverso. A presente casuística de NMCs grandes e gigantes é pequena para avaliar risco de transformação maligna; e o tempo de acompanhamento dos nevos médios e pequenos não é suficiente para avaliação de malignidade, visto que ocorre após a adolescência. 
Tabela 5: Distribuição dos nevos pequenos e médios segundo a localização

\begin{tabular}{|c|c|c|c|c|}
\hline \multirow[b]{2}{*}{ Localização } & \multicolumn{2}{|c|}{ Tipo clínico } & \multirow[b]{2}{*}{ Total } & \multirow[b]{2}{*}{$\%$} \\
\hline & Pequeno & Médio & & \\
\hline Tórax & 15 & 3 & 18 & 27,7 \\
\hline Cabeça e pescoço & 11 & 4 & 15 & 23 \\
\hline Abdômen e virilha & 6 & 6 & 12 & 18,4 \\
\hline Membro inferior & 5 & 6 & 11 & 17 \\
\hline Membro superior & 7 & 1 & 8 & 12,4 \\
\hline Genitália & 1 & 0 & 1 & 1,5 \\
\hline Total & 45 & 20 & 65 & 100 \\
\hline
\end{tabular}

Fonte: IPPMG/UFRJ (1994-2007).

Tabela 6: Distribuição dos nevos melanocíticos segundo tempo de seguimento

\begin{tabular}{|c|c|c|c|c|c|c|c|c|}
\hline \multirow[t]{2}{*}{ Nevos melanocíticos } & \multicolumn{6}{|c|}{ Tempo de seguimento } & \multirow[b]{2}{*}{ Total } & \multirow[b]{2}{*}{$\%$} \\
\hline & $<3$ anos & $\%$ & 3-9 anos & $\%$ & $>10$ anos & $\%$ & & \\
\hline Pequeno & 10 & & 20 & & 15 & & 45 & 60,8 \\
\hline Médio & 5 & & 11 & & 4 & & 20 & 27 \\
\hline Grande & 2 & & 1 & & 1 & & 4 & 5,4 \\
\hline Gigante & 1 & & 3 & & 1 & & 5 & 6,7 \\
\hline Total & 18 & 24,4 & 35 & 47,3 & 21 & 28,3 & 74 & 100 \\
\hline
\end{tabular}

Fonte: IPPMG/UFRJ (1994-2007).

A melanocitose neurocutânea (melanose neurocutânea) é desordem congênita rara na qual a leptomeninge contém quantidades excessivas de melanócitos e melanina; NMC grande/gigante no pescoço, cabeça ou linha média posterior é fator de risco para desenvolvimento da doença. A presença de mais de 20 lesões satélites é outro fator de risco., ${ }^{5,12,24,25}$ Pode ser sintomática ou assintomática; quando sintomática, as manifestações neurológicas surgem antes de dois anos. Dos quatro NMC grandes, apenas um entrou no estudo abaixo de dois anos de idade; dos cinco NMC gigantes, três entraram no estudo até dois anos de idade. Crianças hígidas, assintomáticas, sob o ponto de vista neurológico.

A Ressonância Nuclear Magnética (RNM) tem maior sensibilidade na detecção de melanocitose neurocutânea nas primeiras quatro a seis semanas de vida; como o procedimento requer anestesia geral, é bastante polêmico; à medida que o lactente cresce, tornam-se menos evidentes as pequenas lesões pigmentadas vistas anteriormente. Alguns autores advogam RNM ou exames neurológicos seriados para os pacientes assintomáticos com risco de desenvolverem melanocitose de leptome- ninge. Outros questionam a realização de RNM porque não há tratamento para os casos de melanocitose assintomática. Concordamos com esse questionamento, pois a positividade do exame não alteraria a nossa conduta expectante para nevos grandes/gigantes. ${ }^{12,26}$

São citados como diagnósticos diferenciais dos NMCs, a mancha mongólica, as manchas café-com-leite, o lentigo simples, o nevo epidérmico e o nevo sebáceo, entre os quais, o nevo epidérmico trouxe dificuldade diagnóstica. Pré-escolar, 8 anos, não-branca, com NMC de tamanho médio em região dorsal (caso 26), desenvolveu lesão papulosa, acastanhada, de relevo irregular na região retroauricular direita. O laudo histopatológico da biópsia excisional revelou nevo epidérmico verrucoso. ${ }^{27}$

\section{CONCLUSÃO}

Há divergências quanto à classificação dos NMCs, o que dificulta melhor estudo comparativo. ${ }^{28,29}$

Esta investigação sistematiza, a partir das observações dos pacientes no IPPMG e dos dados de literatura, elementos que podem auxiliar dermatologistas e pediatras no diagnóstico e acompanhamento dos portadores de NMC. 


\section{REFERÊNCIAS}

1. Tannous ZS, Mihm MC Jr, Sober AJ, Duncan LM. Congenital melanocytic nevi: clinical and histopathologic features, risk of melanoma, and clinical management. J Am Acad Dermatol. 2005;52:197-203.

2. Tromberg J, Bauer B, Benvenuto-Andrade C, Marghoob AA. Congenital melanocytic nevi needing treatment. Dermatol Ther. 2005; 18:136-50.

3. Clemmensen J. The histology of "congenital features" in early acquired melanocytic nevi. Dermatology. $1988 ; 19: 742-6$

4. Ruiz-Maldonado R. Measuring congenital melanocytic nevi. Pediatr Dermatol. 2004; 21:178-9.

5. Marghoob AA. Congenital melanocytic nevi: evaluation and management. Dermatol Clin. 2002;20:607-16.

6. Zaal LH, Mooi WJ, Sillevis SJH, Van der Horst CMAM. Classification of congenital melanocytic naevi and malignant transformation: a review of the literature. Br J Plast Surg. 2004;57:707-19.

7. Leech NS, Bell H, Leonard N, Jones SL, Geurin D, Mckee PH, Lawrence CM. Neonatal giant congenital nevi with proliferative nodules. Arch Dermatol. 2004;140:83-8.

8. Esterly NB. Management of congenital melanocytic nevi: a decade later. Pediatr Dermatol. 1996;13:321-40.

9. Rhodes AR, Melski JW. Small congenital nevo cellular nevi and the risk of cutaneous melanoma. J Pediatr. 1982;100:219-24.

10. Krengel S, Hauschild A, Schafert T. Melanoma risk in congenital melanocytic naevi: a systematic review. Br J Dermatol. 2006;155:1-8.

11. Ingordo V, Gentile C, Iannazzone SS, Cusano F, Naldi L. Congenital melanocytic nevus: an epidemiologic study in Italy. Dermatology. 2007;214:227-30.

12. Bett BJ. Large or multiple congenital melanocytic nevi: occurrence of cutaneous melanoma in 1008 persons. J Am Acad Dermatol. 2006;54:767-77.

13. Berg $\mathrm{P}$, Lindelof $\mathrm{B}$. Congenital melanocytic naevi and cutaneous melanoma. Melanoma Res. 2003;13:441-5.

14. West EA, McPartland JL, Rigby H, Parslew RA. Giant bathing trunk naevus with lymphadenopathy and unusual pathology. Br J Dermatol. 2007;157:599-601.

15. Dawson HA, Atherton DJ, Mayou B. A prospective study of congenital melanocytic naevi: progress report and evaluation after 6 years. Br J Dermatol. 1996;134:617-23.

16. Swerdlow AJ, English JSC, Qiao Z. The risk of melanoma in patients with congenital nevocytic nevi: a cohort study. J Am Acad Dermatol. 1995;32:595-99.

17. Arons AS, Hurwitz S. Congenital nevocellular nevus: a review of the treatment controversy and a report of 46 cases. Plast Reconstr Surg. 1983;72:355-65.

18. Castilla EE, Dutra MG, Orioli-Parreiras IM. Epidemiology of congenital pigmented naevi: incidence rates and relative freqüencies. Br J Dermatol. 1981; 104:307-15.
19. Paller AS, Mancini AJ. Hereditary disorders of the dermis. In: Paller AS, Mancini AJ. Hurwitz clinical pediatric dermatology: a textbook of skin disorders of childhood and adolescence. 3rd ed. Philadelphia: Elsevier Saunders, 2006. p.133-4.

20. Zack LD, Stegmeier O, Solomon LM. Pigmentary regression in a giant nevocellular nevus: a case report and a review of the subject. Pediatr Dermatol. 2000;5:178-83.

21. Kageshita T, Inque $Y$, Ono T. Spontaneous regression of congenital melanocytic nevi without evidence of the halo phenomenon. Dermatology. 2003; 207:193-95.

22. Sahin S, Levin H, Kopf AW, Rao BK, Tripola M, Koenig K. Risk of melanoma in medium-sized congenital melanocytic nevi: a follow-up study. J Am Acad Dermatol. 1998; 39:428-33.

23. Maia MR, Ferrari C, Jorge N, Ribeiro D, Muller MC, Helena Giunta, G. Relação entre nevo melanocítico pequeno e melanoma cutâneo. An Bras Dermatol. 2003;78:189-95.

24. Hale EK, Stein J, Ben-Porat L, Panageas K, Eichenbaum M, Marghoob A. Association and neurocutaneous melanocytosis with large congenital melanocytic naevi results from NYU_LCMN registry. $\mathrm{Br} \mathrm{J}$ Dermatol. 2005;152:512-17.

25. Bittencourt FV, Marghoob AA, Kopf AW, Koenig KL, Bart RS. Large Congenital melanocytic nevi and the risk for development of malignant melanoma and neurocutaneous melanocytosis. Pediatrics. 2000;106:36-42.

26. Foster RD, Williams M, Barkovich A, Hoffman W, Mathes S, Frieden I. Giant congenital melanocytic nevi: the significance of neurocutaneous melanosis in neurologically asymptomatic children. Plast Reconstr Surg. 2001;107:933-41.

27. Zitelli JA, Grant MG, Abell E, Boyd JB. Histologic patterns of congenital nevocytic and implications for treatment. J Am Acad Dermatol. 1984;11:402-09.

28. Ingordo V, Gentile C, Iannazzone SS, Cusano F, Naldi L. Congenital melanocytic nevus: an epidmiologic study in Italy. Dermatology. 2007;214:227-30.

29. Schaffer JV. Pigmented lesions in children: when to worry. Curr Opin Pediatr 2007; 19:430-40.

ENDEREÇO PARA CORRESPONDÊNCIA / MAILING ADDRESS:
Nurimar COnceição Fernandes
Rua Alexandre de Gusmão, no 28, Ap. 201
$20520120 \quad$ Rio de Janeiro RJ
Tel./fax: 21 2568-4158
E-mail: nurimarfernandes@terra.com.br

Como citar este artigo / How to cite this article: Fernandes NC, Machado JLR. Estudo clínico dos nevos melanocíticos congênitos na criança e adolescente. An Bras Dermatol. 2009;84(2):129-35. 